\title{
Accès des médecins à la pratique privée
}

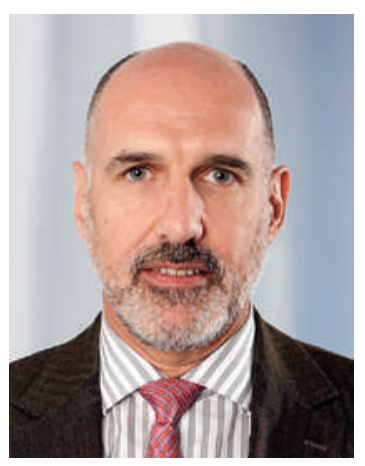

La «clause du besoin» pour les nouveaux cabinets médicaux est un mauvais souvenir... dont on craint un peu, maintenant, qu'il ne laisse la place à un cauchemar: le chaos est en train de s'installer dans le domaine de la démographie médicale.

Après avoir été, malgré toutes les promesses, prolongée deux fois entre 2002 et 2011 - la dernière fois en excluant les médecins de premier recours, mais en incluant les médecins employés dans des institutions ambulatoires - et échue depuis maintenant neuf mois, la clause du besoin n'a guère laissé de souvenirs positifs, c'est le moins qu'on puisse dire.

Echouant bien évidemment à stabiliser les coûts, cette mesure n'a pas non plus stabilisé le nombre de cabinets, ou alors avec des disparités cantonales que même le fédéralisme le plus fervent n'excuse pas. Autre conséquence déplorable, en bloquant les jeunes médecins en milieu hospitalier et en les poussant ainsi à se spécialiser, l'impossibilité d'ouvrir un cabinet a littéralement décapité la relève en médecine de premier recours.

\section{Il est clair pour une majorité des protagonistes qu'une forme de régulation doit être possible - on ne peut pas juste laisser faire!}

Ensuite de quoi, évidemment, la fin du «blocage des cabinets» n'a pas permis la vague espérée d'installations de généralistes, mais a par contre ouvert la porte à une avalanche de demandes de droits de pratique de spécialistes, suisses et étrangers.

Et puis, même si cela ne pouvait d'aucune façon être son but, beaucoup avaient placé dans le projet de loi sur le Managed care l'espoir qu'il aide à réguler la démographie médicale; le cinglant rejet de ce projet de loi a parachevé le sentiment d'impuissance de tous face au nombre de médecins établis, pénurie d'un côté, risque de pléthore de l'autre.

Il est ainsi devenu clair, maintenant, pour une majorité des protagonistes qu'une forme de régulation doit être possible - on ne peut pas juste laisser faire!

Ceci a provoqué un feu d'artifice de propositions de toute sorte chez nos parlementaires: suppression de l'obligation d'assurance, suppression du libre choix du médecin, réintroduction de la clause du besoin, ... chacun, chacune y a été de sa motion!

La situation actuelle de la démographie médicale, c'est-àdire du nombre et de la répartition des médecins, géographi- quement autant que par discipline, n'est effectivement pas satisfaisante, et ce problème a tendance à s'aggraver. On trouve des situations de réelle pénurie, dans plusieurs spécialités; il y a aussi un problème évident de répartition géographique; et enfin, certaines disciplines sont actuellement encore plutôt pléthoriques.

Face à cette situation, la FMH et la Conférence des directeurs sanitaires cantonaux (CDS), en étroite collaboration, ont réactivé une excellente proposition [1] qu'elles avaient déjà faite en commun, en 2006 et en 2008, et qui avait été enterrée par le Parlement de l'époque.

\section{La régulation ne peut s'exercer qu'exceptionnellement, si nécessaire, et elle est alors extrêmement flexible.}

En quoi consiste cette proposition?

Elle prévoit, en cas de besoin démontré par des chiffres, de donner explicitement aux cantons la possibilité de gérer la démographie médicale, par discipline et par région - autrement dit, la régulation ne peut s'exercer qu'exceptionnellement, si nécessaire, et elle est alors extrêmement flexible; elle peut se faire soit au moyen d'incitatifs, soit en jouant sur l'attribution des droits de pratique.

Pour rester en résonnance avec «le terrain», il est prévu que tous les partenaires (médecins, y compris les assistants et chefs de clinique, hôpitaux, assureurs) soient entendus lorsque des mesures sont prises.

Autre élément important et innovant: le droit de pratique peut être soumis à une expérience réelle, concrète, du système de santé suisse et à la connaissance de la langue. Cela nous semble nécessaire à un bon accompagnement des patients, c'est-à-dire à une médecine de qualité; selon notre projet, on peut exiger d'avoir travaillé trois ans en Suisse pour ouvrir un cabinet.

Reste à espérer que cette proposition de la FMH et de la CDS trouvera grâce devant le Parlement, cette fois - les autres «solutions» envisagées actuellement ne sont en réalité pas acceptables: le libre choix du médecin est intangible, tant aux yeux du corps médical que pour la population, on l'a vu le 17 juin dernier; et réintroduire la «clause du besoin» serait vraiment une honte.

Nous allons donc nous engager pour faire aboutir notre projet, qui est indubitablement convaincant.

Dr Jacques de Haller, Président de la FMH

1 www.fmh.ch $\rightarrow$ POLITIQUE\&MEDIAS $\rightarrow$ Dossiers $\rightarrow$ Régulation des ressources 